\title{
Radioactive Iodine Ablation Decrease Recurrences in Papillary Thyroid Microcarcinoma with Lateral Lymph Node Metastasis in Chinese Patients
}

\author{
Shuai Xue ${ }^{1} \cdot$ Peisong Wang ${ }^{1} \cdot$ Jia Liu $^{1} \cdot$ Guang Chen $^{1}$
}

Published online: 24 July 2017

(C) The Author(s) 2017. This article is an open access publication

\begin{abstract}
Introduction We aimed to carry out a retrospective study from a single institution to determine whether radioactive iodine (RAI) ablation decreases the risk of recurrence of papillary thyroid microcarcinoma (PTMC) patients that presented with lateral lymph node metastasis (LLNM).

Methods We retrospectively analyzed a total of 6361 consecutive PTMC patients who initially underwent surgery for the treatment of thyroid carcinoma at the First Hospital of Jilin University, between January 2005 and February 2015. Altogether, 137 patients with PTMC with LLNM have been enrolled in our study.

Results The disease-free survival period was significantly shorter for the RAI $(-)$ patients than for the RAI $(+)$ patients ( $p=0.0074$ by the log-rank test). The disease-free survival rates at 5 and 10 years were 93.02 and $87.5 \%$, respectively, in the RAI (-) group and 100 and $96.81 \%$, respectively, in the RAI (+) group. CLNM ratio and LLNM ratio were factors identified for multivariate analysis by Cox's proportional hazards method yielding risk ratios of 7.281 [CI 1.804-17.554; $p=0.010$ ] and 1.157 [CI 1.0125-9.381; $p=0.048$ ] in the RAI $(-)$ group.

Conclusion Taken together, RAI may be beneficial for PTMC with LLNM, especially when CLNM ratio or LLNM ratio was greater than 0.5 .
\end{abstract}

\section{Introduction}

Papillary thyroid carcinoma (PTC) is the most common type of endocrine malignant cancers. Owing to a rapid increase in diagnosis of papillary thyroid microcarcinoma (PTMC), the incidence of PTC has been also increasing sharply [1]. This increase in PTMC resulted mainly from the increased detection of small nonpalpable nodules by high-frequent ultrasound and fine needle aspiration [2]. It has been reported that $38.5 \%$ among PTC in the USA were PTMC, while $48.8 \%$ in France and $35.7 \%$ in Shanghai, China $[3,4]$.

Guang Chen

jidayiyuanjzx@sina.com

1 Department of Thyroid Surgery, The 1st Hospital of Jilin University, Changchun 130021, People's Republic of China
On the basis of the American Thyroid Association (ATA) guidelines in 2015, radioactive iodine (RAI) ablation was recommended for high-risk patients with gross extrathyroidal extension (ETE), distant metastasis, or incomplete tumor resection. For unifocal and multifocal PTMC without other adverse features, RAI was not routinely recommended. Because of low-quality evidence and the lack of long-term prognosis analysis, the guidelines just made week recommendation for intermediate-risk differentiated thyroid carcinoma [5].

Regarding to PTMC, several researchers found that PTMC, which had the characteristics of a malignant cancer, was often multifocal and prone to central lymph node metastasis (CLNM) [6-8]. Therefore, they suggested aggressive operation and even RAI ablation postoperatively for PTMC patients. In cases of differentiated thyroid carcinoma with LLNM, RAI was recommended by ATA 
because of the higher risk of persistent or recurrent disease, especially with the presence of extra-nodal extension or clinically positive or increasing number of macroscopic lymph nodes [5]. However, increasing studies demonstrated that the vast majority of PTMC were inert carcinomas, so aggressive surgery and RAI ablation were considered as overtreatments because they could not improve the recurrence and outcome [9, 10]. Moreover, such systematic review and meta-analysis of the effectiveness of RAI therapy for PTMC have been performed and conclude that for PTMC patients already treated by total thyroidectomy (TT), RAI ablation may not be helpful to decrease the incidence of thyroid cancer-related mortality or the 10-year recurrence of PTMC. Even for the intermediate-risk PTMC patients, RAI did not prevent recurrence [11]. Based on these disputes, the efficacy of RAI therapy for PTMC with lateral lymph node metastases (LLNM) is still unknown.

Thus, we aimed to carry out a retrospective study from a single institution to discuss whether RAI ablation decreases the risk of recurrence of PTMC patients that presented with LLNM.

\section{Materials and methods}

\section{Patients}

This study was approved by the Ethical Committee of the First Hospital of the Jilin University, and written informed consents were given to participants for their clinical records to be used in this study. We retrospectively analyzed a total of 6361 consecutive PTMC patients who initially underwent surgery for the treatment of thyroid carcinoma at the First Hospital of Jilin University, between January 2005 and February 2015. One hundred and thirtyseven patients recruited in the study met the following criteria: (a) patients' information found in a hospital database and (b) patients with a postoperative pathological diagnosis of PTMC with LLNM. (c) Patients underwent total thyroidectomy with bilateral central lymph node dissection (CLND) and unilateral or bilateral lateral lymph node dissection (LLND). Patients were excluded from the study if pathological types of thyroid malignancies other than PTMC, age $<18$ years, high-risk patients with gross extrathyroidal extension (ETE), distant metastasis or incomplete tumor resection, lack of a preoperative fine needle aspiration (FNA) examination, had a history of neck radiotherapy, and with history of previous thyroid surgery, follow-up duration $<6$ months (loss to follow-up within 6 months, reoperation within 6 months after initial surgery, or residual malignancy or lymph node detected within
6 months after initial surgery). Finally, this study enrolled 137 patients with PTMC with LLNM.

\section{Initial treatment}

Clinical diagnosis was initially made by examination of ultrasound (US) and FNA. US cervical mapping was done by an experienced, specially trained radiologist. For PTMC with LLNM, we performed TT and bilateral CLND with unilateral or bilateral LLND. This study included cases with both prophylactic and therapeutic CLND. CLND was performed to remove all lymph nodes and fibro-fatty tissue extending laterally from the medial border of the common carotid artery to the midline of the trachea and vertically from the hyoid bone to the thoracic inlet. All the patients underwent therapeutic LLND due to preoperative confirmation of LLNM which was diagnosed by US, CT, and/or FNA. In our institution, modified LLND including levels II-V with the preservation of the spinal accessory nerve, internal jugular vein, and sternocleidomastoid muscle is the standard treatment for LLNM. Level I dissection was not performed routinely unless indicated.

\section{Clinicopathological variable}

Demographic data on patient clinical characteristics such as gender and age at diagnosis, as well as histopathological features (diameter of the largest tumor, total diameter of all tumors, multifocality, bilaterality, variants of PTMC, v-Raf murine sarcoma viral oncogene homolog B (BRAF) mutation, microscopic ETE, with chronic thyroiditis, regional lymph node metastasis, total and metastatic lymph node yield, TSH suppression and administered RAI), were recorded.

\section{Follow-up}

Until 2011, TSH-suppressive hormonal therapy was applied to postoperative patients. We followed up all cases with physical examinations, serum thyroglobulin $(\mathrm{Tg}), \mathrm{Tg}$ antibodies, US, and iodine-131 scans at 6-month intervals. There are no definite benefits that PTMC patients with LLNM could obtain from RAI ablation. As a result, surgeons must explain both the risks and benefits of RAI ablation and give informed consent forms to patients. The final decision was made based on physician or patient preference. When recurrence was suspected, patients underwent FNA with or without measurement of washout Tg levels and thyroid CT. In our study, recurrence was defined as the presence of tumor or metastatic lymph node at least 6 months after the initial surgery. 
Table 1 Clinicopathological characteristics of PTMC patients with LLNM according to RAI ablation

$\begin{array}{ll}\text { RAI }(-) & \text { RAI }(+) \\ (N=43) & (N=94)\end{array}$

$p$ value

$(N=43) \quad(N=94)$

Gender

Female

30

13

71

Male

Age

$<45 \mathrm{yr}$

21

$\geq 45 \mathrm{yr}$

34

73

0.853

Diameter of largest tumor

$\leq 0.5 \mathrm{~cm}$

14

19

$>0.5 \mathrm{~cm}$

29

75

0.250

Total diameter of all tumors

$\leq 0.5 \mathrm{~cm}$

2

6

$0.5-1.0 \mathrm{~cm}$

14

18

$1.0-2.0 \mathrm{~cm}$

11

34

$>2.0 \mathrm{~cm}$

16

36

0.329

Multifocality

Absent

22

Present

Bilaterality

Absent

35

Present

Microscopic ETE

Absent

3

Present

40

With chronic thyroiditis

Absent

Present

Variants of PTMC

Classical

Follicular

Diffuse sclerosing

2

Tall cell

Solid

Insular

Oxyphilic

Columnar

Others

2

0

1

1

0

0

0

0

0.608

BRAF mutation

Absent

6

Present

37

16

78

0.650

LLND

Unilateral

40

86

Bilateral

3

Number of total CLN

$5.17 \pm 1.02$

8

1.000

$1.43 \pm 0.09$

$6.01 \pm 1.14$

0.998

$11.2 \pm 2.11$

0.223

Number of total LLN

$4.93 \pm 1.11$

$2.82 \pm 0.77$

1.000

Number of metastatic LLN

$10.98 \pm 2.07$

0.573

CLNM ratio 
Table 1 continued

\begin{tabular}{|c|c|c|c|}
\hline & $\begin{array}{l}\text { RAI }(-) \\
(N=43)\end{array}$ & $\begin{array}{l}\text { RAI }(+) \\
(N=94)\end{array}$ & $p$ value \\
\hline$\geq 0.5$ & 10 & 15 & 0.305 \\
\hline \multicolumn{4}{|l|}{ LLNM ratio } \\
\hline$<0.5$ & 34 & 81 & \\
\hline$\geq 0.5$ & 9 & 13 & 0.294 \\
\hline \multicolumn{4}{|l|}{ TSH supperssion } \\
\hline Yes & 37 & 85 & \\
\hline No & 6 & 9 & 0.446 \\
\hline Average follow-up period (years) & $6.75 \pm 2.11$ & $6.07 \pm 1.97$ & 0.816 \\
\hline \multicolumn{4}{|l|}{ Administered RAI } \\
\hline $0 \mathrm{mCi}$ & 43 & 0 & \\
\hline$\leq 30 \mathrm{mCi}$ & 0 & 2 & \\
\hline $30-100 \mathrm{mCi}$ & 0 & 15 & \\
\hline $100-150 \mathrm{mCi}$ & 0 & 59 & \\
\hline$>150 \mathrm{mCi}$ & 0 & 18 & 0.000 \\
\hline
\end{tabular}

ETE extrathyroidal extension, LLND lateral lymph node dissection, PTMC papillary thyroid microcarcinoma, CLNM ratio central lymph node metastasis ratio (positive lymph node number/sum lymph node number), LLNM ratio lateral lymph node metastasis ratio (positive lymph node number/sum lymph node number)

\section{Statistical analysis}

To identify differences between groups for specific variables, SPSS version 22 software (SPSS Inc, Chicago, IL) was used for statistical analysis, which was performed by Pearson's Chi-square test or Student's $t$-test. Survival curves were drawn by Kaplan-Meier method and statistically analyzed by the log-rank test. To characterize PTMC with LLNM, univariate analysis and multivariate analysis were performed by Cox's proportional hazards method for disease-free survival and the risk factors. A $p$ value $<0.05$ was considered statistically significant.

\section{Results}

Altogether, 137 patients of PTMC with LLNM have been enrolled in our study, in which 94 cases underwent with RAI while other 43 cases did not. The clinicopathological characteristics of the enrolled patients $(n=137)$ are shown in Table 1. Administered RAI was found to be different significantly between two groups $(p=0.000)$. There were no significant differences between the two groups in terms of age, gender, microscopic ETE, diameter of the largest tumor, total diameter of all tumors, multifocality, bilaterality, presence of chronic thyroiditis, variants of PTMC, BRAF mutation, LLND, total and metastatic lymph node yield, CLNM ratio, LLNM ratio, and TSH suppression.

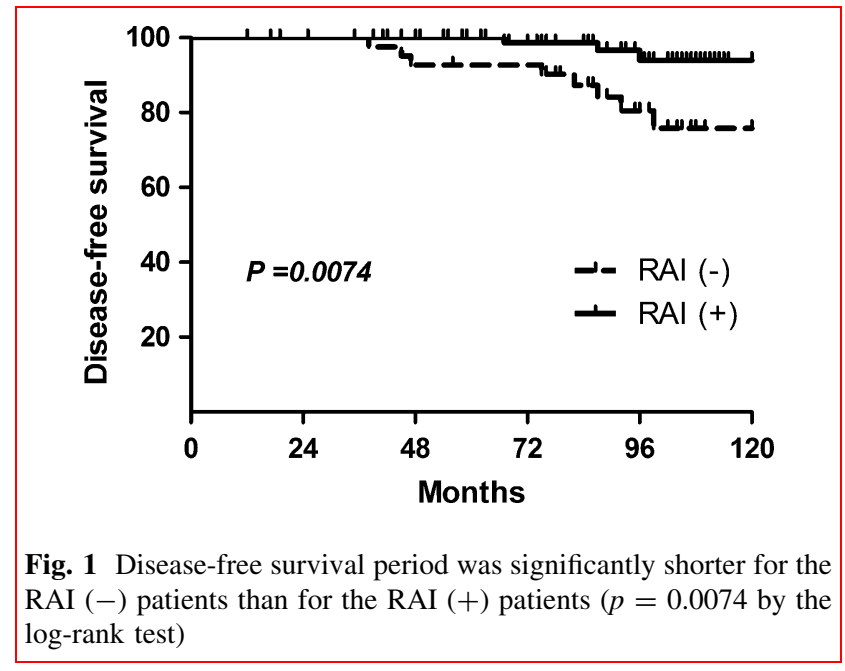

The disease-free survival period was significantly shorter for the RAI $(-)$ patients than for the RAI $(+)$ patients $(p=0.0074$ by the log-rank test) (Fig. 1). Details of recurrent cases are shown in Table 2. The disease-free survival rates at 5 and 10 years were 93.02 and $87.5 \%$, respectively, in the RAI (-) group and 100 and $96.81 \%$, respectively, in the RAI $(+)$ group. Because nobody died of thyroid cancer, disease-specific mortality did not differ statistically between groups (data not shown).

Univariate analysis by Cox's proportional hazards method showed CLNM ratio and LLNM ratio to be risk factors for recurrence of RAI (-) patients (Table 3). Age, 


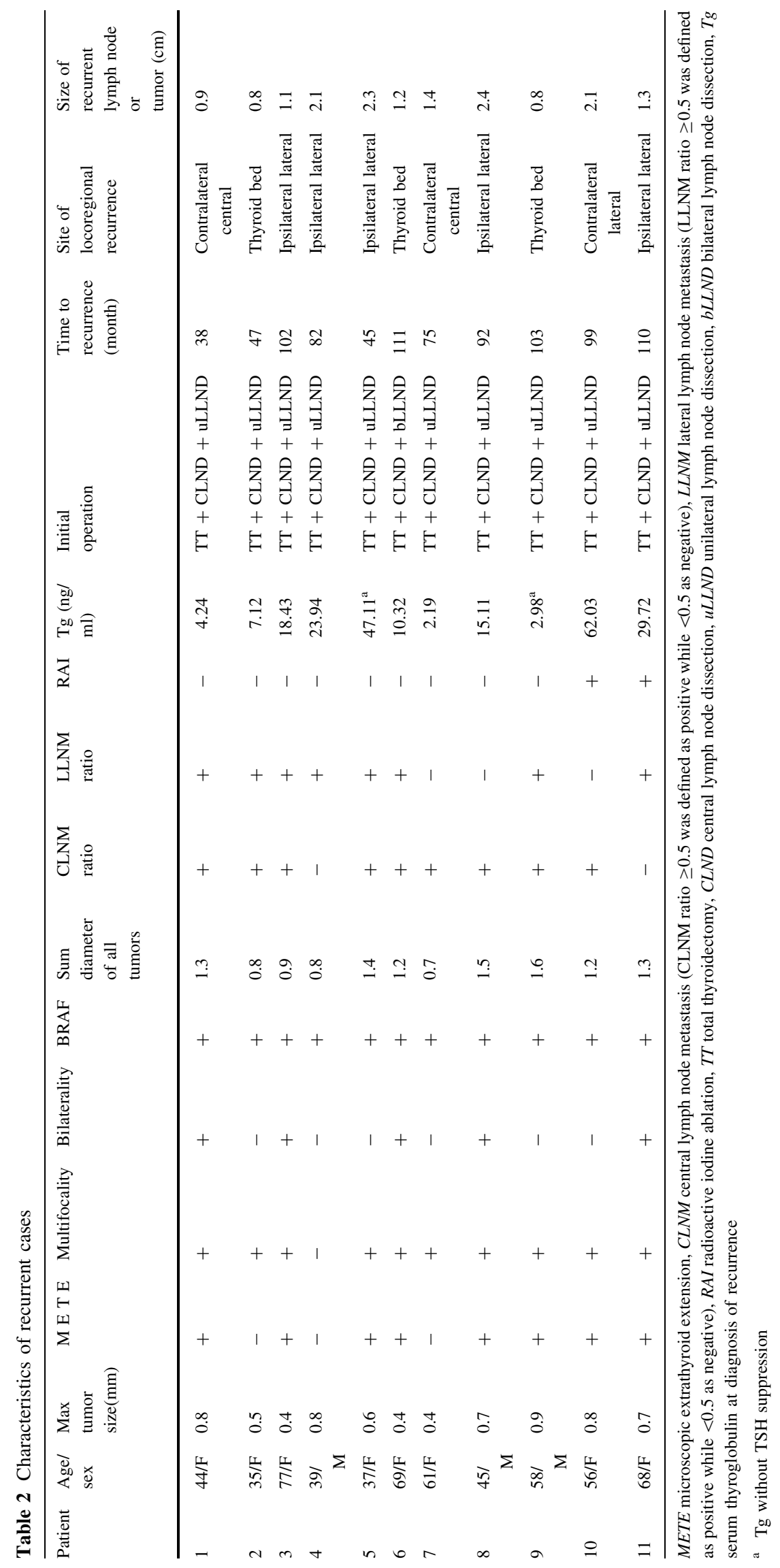


Table 3 Subgroup univariate analysis by Cox's proportional hazards method for disease-free survival

\begin{tabular}{lcc}
\hline Factors analyzed & \multicolumn{2}{l}{ RAI $(-)$ patients } \\
\cline { 2 - 3 } & Risk ratio (CI) & $p$ value \\
\hline Age (years) & $0.034(0.000-48.227)$ & 0.362 \\
Male gender & $1.170(0.279-4.911)$ & 0.830 \\
Microscopic ETE & $0.794(0.096-6.551)$ & 0.830 \\
Diameter of largest tumor & $0.621(0.148-2.609)$ & 0.515 \\
Sum diameter of all tumors & $1.624(0.388-6.802)$ & 0.507 \\
LLND bilaterality & $2.070(0.254-16.892)$ & 0.497 \\
Multifocality & $1.051(0.211-5.239)$ & 0.952 \\
Bilaterality & $0.443(0.106-1.855)$ & 0.265 \\
With chronic thyroiditis & $0.895(0.109-7.360)$ & 0.918 \\
BRAF mutation & $1.443(0.177-11.780)$ & 0.732 \\
Variants of PTMC & $0.595(0.073-4.842)$ & 0.628 \\
CLNM Ratio & $12.948(2.610-64.229)$ & 0.002 \\
LLNM Ratio & $8.321(1.234-32.321)$ & 0.031
\end{tabular}

ETE extrathyroidal extension, $L L N D$ lateral lymph node dissection, PTMC papillary thyroid microcarcinoma, CLNM ratio central lymph node metastasis ratio (metastatic central lymph node/harvested central lymph node), LLNM ratio lateral lymph node metastasis ratio (metastatic lateral lymph node/harvested lateral lymph node)

gender, microscopic ETE, diameter of the largest tumor, total diameter of all tumors, multifocality, bilaterality, presence of chronic thyroiditis, variants of PTMC, BRAF mutation, and LLND were not predictors of recurrence. The risk ratio for CLNM ratio and LLNM ratio was 12.948 [confidence interval (CI) 2.610-64.229; $p=0.002$ ] and 8.321 [CI 1.234-32.321; $p=0.031$ ] in the RAI $(-)$ group. No other risk ratios obtained were of interest. CLNM ratio and LLNM ratio were factors identified for multivariate analysis by Cox's proportional hazards method (Table 4) yielding risk ratios of 7.281 [CI 1.804-17.554; $p=0.010$ ] and 1.157 [CI $1.0125-9.381 ; p=0.048$ ] in the RAI $(-)$ group. The disease-free survival period was significantly shorter for the CLNM ratio $\geq 0.5$ and LLNM ratio $\geq 0.5$ patients $(p<0.0001$ and $p=0.0158$ by the log-rank test)

Table 4 Subgroup multivariate analysis by Cox's proportional hazards method for disease-free survival

\begin{tabular}{lll}
\hline Factors analyzed & \multicolumn{2}{l}{ RAI $(-)$ patients } \\
\cline { 2 - 3 } & Risk ratio $(\mathrm{CI})$ & $p$ value \\
\hline CLNM ratio & $7.281(1.804-17.554)$ & 0.010 \\
LLNM ratio & $1.157(1.0125-9.381)$ & 0.048 \\
\hline
\end{tabular}

CLNM ratio central lymph node metastasis ratio (metastatic central lymph node/harvested central lymph node), LLNM ratio lateral lymph node metastasis ratio (metastatic lateral lymph node/harvested lateral lymph node)

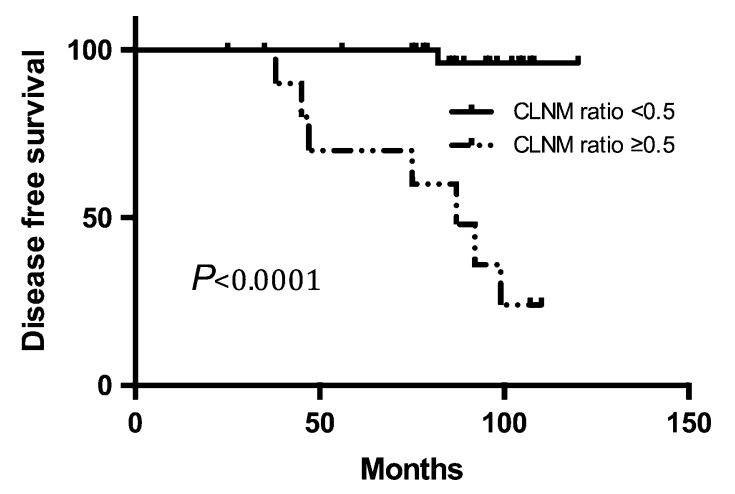

Fig. 2 Disease-free survival period was significantly shorter for the CLNM ratio $\geq 0.5$ patients than for the CLNM ratio $<0.5$ patients $(p<0.0001$ by the log-rank test $)$

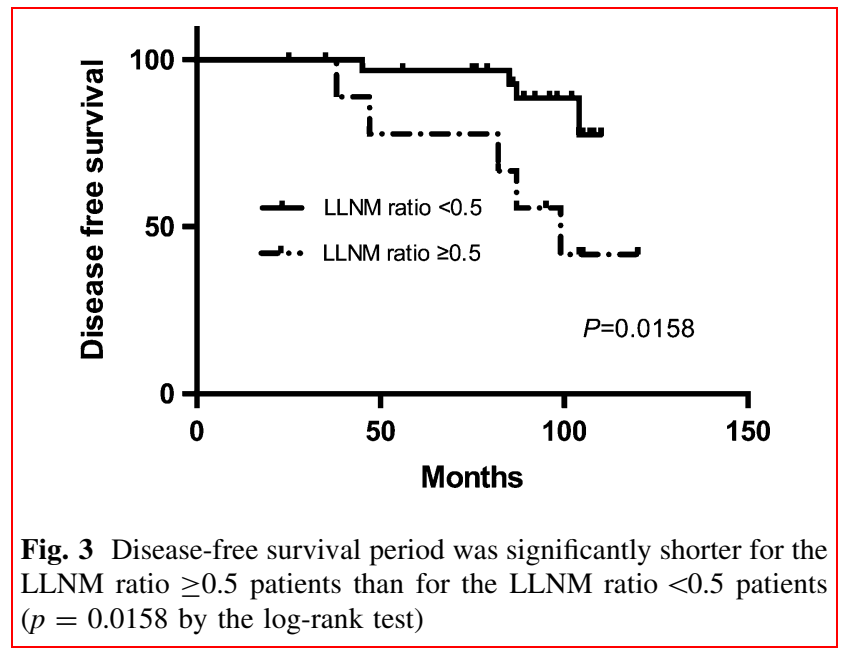

(Figs. 2, 3). Accordingly, CLNM ratio and LLNM ratio were shown to be independent predictors of disease-free survival.

\section{Discussion}

There is a wide awareness that radioactive treatment can eliminate residual normal or tumor thyroid cells which further leads to decrease recurrence [12]. Although generally safe, RAI has several potential side effects, classified as early and late complications [13, 14]. More severely, when compared to the general population, female PTC patients who treated with RAI had increasing risk of second primary malignancy, and this risk was not apparent significantly in those without RAI $[15,16]$. The medical decision process for referral to treatment with RAI should therefore incorporate a careful analysis of the benefits and risks for this therapy, particularly in the case of younger patients. Moreover, increasing reports published recently have demonstrated that RAI could not decrease disease- 
free survival and disease-specific mortality in PTMC patients, even in the intermediate-risk DTC [10, 11]. Thus, we performed this retrospective study to evaluate the function of RAI in PTMC with LLNM (belong to intermediated-risk DTC) and explore the prognostic factors for recurrence.

In our study, the disease-free survival period was significantly shorter for the RAI (-) patients than for the RAI $(+)$ patients $(p=0.0074$ by the log-rank test). Creach et al. [17] reviewed the therapy and outcome of 407 patients with PTMC, and they found that the 5-year disease-free survival for patients treated with RAI was 95.0 versus $78.6 \%$ $(p<0.0001)$ for patients not treated with RAI. In their study, patients with lymph node metastasis who did not receive RAI had a 5-year disease-free survival of 42.9 versus $93.2 \%(p<0.0001)$ for patients who received RAI, which was consistent with our research. Moreover, ALQahtani et al. [18] concluded in their study that adjuvant RAI therapy improves disease-free survival in PTMC patients with aggressive histopathologic variants, multifocality, ETE, lymphovascular space invasion, tumor size $(>0.5 \mathrm{~cm})$, and lymph node involvement. However, in recent years, several studies suggested that RAI did not prevent recurrences in patients with PTMC. In Hye Jeong Kim's study, we have huge concerns about the selection bias since the RAI (-) group was only 30 cases compared with the RAI (+) group of 450 patients in the subgroup of intermediated risk [10]. Furthermore, $74 \%$ cases in RAI (-) group were TNM stage I while only $47 \%$ in RAI (+) group. In another systemic review and meta-analysis, they found that there was almost no positive treatment effect of RAI ablation noted for patients with PTMC. But the dose of RAI was unavailable in most studies and the follow-up period was different among researches. Consequently, these conclusions were not powerful enough to demonstrate efficacy of RAI in PTMC.

Massive prognostic parameters for recurrence of PTMC have been identified in previous studies, such as male gender, age $<45$, ETE, multifocality, primary tumor size, BRAF, T3 tumor, and lymph node metastasis [19-27]. In our subgroup study analysis, CLNM ratio and LLNM ratio were factors identified for multivariate analysis by Cox's proportional hazards method (Table 4 ) yielding risk ratios of 7.281 [CI 1.804-17.554; $p=0.010$ ] and 1.157 [CI $1.0125-9.381 ; p=0.048]$ in the RAI $(-)$ group. The disease-free survival period was significantly shorter for the CLNM ratio $\geq 0.5$ and $L L N M$ ratio $\geq 0.5$ patients ( $p<0.0001$ and $p=0.0158$ by the log-rank test) (Figs. 2, $3)$. Accordingly, CLNM ratio and LLNM ratio were shown to be independent predictors of disease-free survival. Like a previous study, when the CLNM ratio is higher than 0.44 , there is an increased risk of locoregional recurrence mostly in the lateral neck [21]. Additionally, Kim et al. [25] also found LLNM was a risk factor for recurrent PTMC. Accordingly, we believe that RAI may be beneficial for PTMC with LLNM, especially when CLNM ratio or LLNM ratio was greater than 0.5 .

Our study has some limitations. The first limitation is the small number of patients in the study population; Due to the incidence of PTMC with LLNM is low, and we want long duration of follow-up, we just summarized patients between January 2005 and February 2015. The second limitation is that until 2011, TSH-suppressive hormonal therapy was applied to postoperative patients. At that time, there was no guideline which we could follow to recommend TSH-suppressive hormonal in China. Despite these limitations, our study has important implications for PTMC management and provides significant information for PTMC guideline formation.

\section{Conclusion}

Taken together, RAI may be beneficial for PTMC with LLNM, especially when CLNM ratio or LLNM ratio was greater than 0.5. A multi-centric pragmatic randomized controlled clinical trial with large population is needed to assess the effects on recurrence, quality of life, and costeffectiveness outcomes of different treatments and followup regimen for PTMC with LLNM.

Acknowledgements I would like to show my deepest gratitude to my supervisor, Prof. Feng Guan, a respectable, responsible, and resourceful scholar in Nuclear Medicine, who has provided me with valuable guidance in every stage of the writing of this thesis. Without his enlightening instruction, impressive kindness and patience, I could not have completed my thesis. His keen and vigorous academic observation enlightens me not only in this thesis but also in my future study.

Authors' contributions Shuai Xue, Jia Liu, and Peisong Wang participated in the design of the study and performed the statistical analysis. Guang Chen conceived of the study, participated in its design and coordination, and helped to draft the manuscript. All authors read and approved the final manuscript.

\section{Compliance with ethical standards}

Conflict of interest The authors declare that they have no competing interests.

Open Access This article is distributed under the terms of the Creative Commons Attribution 4.0 International License (http://crea tivecommons.org/licenses/by/4.0/), which permits unrestricted use, distribution, and reproduction in any medium, provided you give appropriate credit to the original author(s) and the source, provide a link to the Creative Commons license, and indicate if changes were made. 


\section{References}

1. Hedinger C, Wiliams ED, Sobin LH (1988) Histologic typing of thyroid tumors. International histological classification of tumors. No.11. World Health Organization. Springer, Berlin, pp 1-18

2. Wang TS, Goffredo P, Sosa JA et al (2014) Papillary thyroid microcarcinoma: an over-treated malignancy? World J Surg 38:2297-2303. doi:10.1007/s00268-014-2602-3

3. Davies L, Welch HG (2006) Increasing incidence of thyroid cancer in the United States, 1973-2002. Jama 295:2164-2167

4. Baudin E, Travagli JP, Ropers J et al (1998) Microcarcinoma of the thyroid gland: the Gustave-Roussy Institute experience. Cancer 83:553-559

5. Haugen BR, Alexander EK, Bible KC et al (2016) 2015 American Thyroid Association management guidelines for adult patients with thyroid nodules and differentiated thyroid cancer: the American Thyroid Association Guidelines task force on thyroid nodules and differentiated thyroid cancer. Thyroid 26(1):1-133

6. Park JP, Roh J-L, Lee JH et al (2014) Risk factors for central neck lymph node metastasis of clinically noninvasive, node negative papillary thyroid microcarcinoma. Am J Surg 208:412-418

7. Yang Y, Chen C, Chen Z et al (2014) Prediction of central compartment lymph node metastasis in papillary thyroid microcarcinoma. Clin Endocrinol 81:282-288

8. Zhang L, Wei W-J, Ji Q-H et al (2012) Risk factors for neck nodal metastasis in papillary thyroid microcarcinoma: a study of 1066 patients. J Clin Endocrinol Metab 97:1250-1257

9. Kim HJ, Kim NK, Choi JH et al (2013) Radioactive iodine ablation does not prevent recurrences in patients with papillary thyroid microcarcinoma. Clin Endocrinol 78:614-620

10. Kim SK, Woo J-W, Lee JH et al (2016) Radioactive iodine ablation may not decrease the risk of recurrence in intermediaterisk papillary thyroid carcinoma. Endocr Relat Cancer 23(5):367-376

11. Hu G, Zhu W, Yang W et al (2016) The effectiveness of radioactive iodine remnant ablation for papillary thyroid microcarcinoma: a systematic review and meta-analysis. World J Surg 40:100-109. doi:10.1007/s00268-015-3346-4

12. Patel SS, Goldfarb M (2012) Well-differentiated thyroid carcinoma: the role of post-operative radioactive iodine administration. J Surg Oncol 107:665-672

13. Rosario PW, Calsolari MR (2013) Salivary and lacrimal gland dysfunction after remnant ablation with radioactive iodine in patients with differentiated thyroid carcinoma prepared with recombinant human thyrotropin. Thyroid 23(5):617-619

14. Fard-Esfahani A, Emami-Ardekani A, Fallahi B et al (2014) Adverse effects of radioactive iodine-131 treatment for differentiated thyroid carcinoma. Nucl Med Commun 35:808-817
15. Piciu D, Pestean C, Barbus E et al (2016) Second malignancies in patients with differentiated thyroid carcinoma treated with low and medium activities of radioactive I-131. Clujul Med 89(3):384-389

16. Lang BH-H, Wong IOL, Wong KP et al (2012) Risk of second primary malignancy in differentiated thyroid carcinoma treated with radioactive iodine therapy. Surgery $151: 844-850$

17. Creach KM, Siegel BA, Nussenbaum B et al (2012) Radioactive iodine therapy decreases recurrence in thyroid papillary microcarcinoma. ISRN Endocrinol. doi:10.5402/2012/816386

18. AL-Qahtani KH, Al Asiri M, Tunio MA et al (2015) Adjuvant radioactive iodine 133 ablation in papillary microcarcinoma of thyroid: Saudi Arabian experience. J Otolaryngol-Head Neck Surg 44:51-60

19. Pyo JS, Sohn JH, Kang G (2016) Detection of tumor multifocality is important for prediction of tumor recurrence in papillary thyroid microcarcinoma: a retrospective study and meta-analysis. J Pathol Transl Med 50:278-286

20. Mehanna H, Al-maqbili T, Carter B (2014) Differences in the recurrence and mortality outcomes rates of incidental and nonincidental papillary thyroid microcarcinoma: a systematic review and meta-analysis of 21329 person-years of follow-up. J Clin Endocrinol Metab 99(8):2834-2843

21. Choi SY, Cho J-K, Moon JH et al (2016) Metastatic lymph node ratio of central neck compartment has predictive values for locoregional recurrence in papillary thyroid microcarcinoma. Clin Exp Otorhinolaryngol 9(1):75-79

22. Pisanu A, Saba A, Podda M et al (2015) Nodal metastasis and recurrence in papillary thyroid microcarcinoma. Endocrine 48:575-581

23. Lombardi CP, Bellantone R, De Crea C et al (2010) Papillary thyroid microcarcinoma: extrathyroidal extension, lymph node metastases, and risk factors for recurrence in a high prevalence of goiter area. World J Surg 34:1214-1221. doi:10.1007/s00268009-0375-x

24. Pisanu A, Reccia I, Nardello O et al (2009) Risk factors for nodal metastasis and recurrence among patients with papillary thyroid microcarcinoma: differences in clinical relevance between nonincidental and incidental tumors. World J Surg 33:460-468. doi:10.1007/s00268-008-9870-8

25. Kim TY, Hong SJ, Kim JM et al (2008) Prognostic parameters for recurrence of papillary thyroid microcarcinoma. BMC Cancer 8:296

26. Siddiqui $\mathrm{S}$, White MG, Antic T et al (2016) Clinical and pathologic predictors of lymph node metastasis and recurrence in papillary thyroid microcarcinoma. Thyroid 26(6):807-815

27. Chen Y, Sadow PM, Suh H et al (2016) BRAFV600E is correlated with recurrence of papillary thyroid microcarcinoma: a systematic review, multi-institutional primary data analysis, and meta-analysis. Thyroid 26(2):248-255 